Research Paper

\title{
Copper Nanoparticles Show Obvious in vitro and in vivo Reproductive Toxicity via ERK Mediated Signaling Pathway in Female Mice
}

\author{
Cai-Hong Zhang ${ }^{1 *}$, Ye Wang ${ }^{2,3^{*}}$,Qian-Qian Sun ${ }^{1,4^{*}}$, Lei-Lei Xia ${ }^{1}$, Jing-Jing Hu${ }^{5}$, Kai Cheng ${ }^{5}$, Xia Wang ${ }^{6}$, \\ Xin-Xin $\mathrm{Fu}^{7}$, Hang $\mathrm{Gu}^{1 凶}$
}

1. Department of Obstetrics and Gynaecology, Changhai Hospital, Second Military Medical University, Shanghai 200433, People's Republic of China,

2. Department of Cell Biology, Second Military Medical University, Shanghai, People's Republic of China,

3. Department of Urology, Changhai Hospital, Second Military Medical University, Shanghai, People's Republic of China,

4. Department of Gynaecology, Shanghai First Maternity and Infant Hospital, Tongji University School of Medicine, Shanghai 200040, People's Republic of China,

5. Clinical Research Center, Changhai Hospital of Second Military Medical University, Shanghai 200433, China,

6. Department of Medical Information, Second Military Medical University, Shanghai, People's Republic of China,

7. Department of Nursing, Second Military Medical University, Shanghai, People's Republic of China.

*Authors contributed equally.

$\square$ Corresponding author: Hang Gu, Department of Obstetricsand Gynecology, Changhai Hospital, Second Military Medical University,168 Changhai Road, Shanghai, 200433, China. Tel: +86-21- 81870407; Fax: +86-21- 81870407; E-mail: guhh@sina.com

(c) Ivyspring International Publisher. This is an open access article distributed under the terms of the Creative Commons Attribution (CC BY-NC) license (https://creativecommons.org/licenses/by-nc/4.0/). See http://ivyspring.com/terms for full terms and conditions.

Received: 2018.06.04; Accepted: 2018.09.02; Published: 2018.10.20

\begin{abstract}
Copper nanoparticles (Cu-NPs) and other inorganic nanomaterials have caused increasing concern owing to be widely used. Early studies have reported that they can result in injuries to the kidney, liver and spleen of mice; cause embryonic damage; and inhibit the reproductive capacity of red worms. However, few studies have reported the toxicity of Cu-NPs on the reproductive systems of mammals. In the present work, we explored the cytotoxicity of Cu-NPs in human extravillous trophoblast cells and in the reproductive organs of mice. Cu-NPs induced ovarian and placental pathophysiology and dysfunction in mice. These nanoparticles also induced apoptosis and suppressed the proliferation of human extravillous trophoblast cells and caused cell cycle arrest at the $\mathrm{G} 2 / \mathrm{M}$ phase in a time-and dose-dependent manner. Cu-NPs can significantly damage the mitochondrial membrane potential (MMP), which suggests that $\mathrm{Cu}-\mathrm{NPs}$ can activate the mitochondria-mediated apoptosis signaling pathway. We also observed that Cu-NPs significantly inhibit the expression of BRAF, ERK, and MITF expression, all of which are important genes in the ERK signaling pathway. Our research demonstrated that Cu-NPs exert obvious reproductive toxicity in mice by disrupting the balance of sex hormones and exert cytotoxicity on human extravillous trophoblast cells, and ERK signaling and the mitochondrial apoptosis pathway made great contribution to the toxicity of Cu-NPs on female mice.
\end{abstract}

Key words: Cu-NPs, human extravillous trophoblast cell, ERK mitochondria membrane potential, reproductive toxicity

\section{Introduction}

With the rapid developments in nanoscience and nanotechnology in recent years, various types of nanomaterials have been widely applied in areas of medicine, biology and food manufacturing because of the unique physicochemical properties and innovative function of these materials [1-3]. Many nanomaterials naturally exist within environmental pollution and can also be either produced or engineered intentionally [4]. Although the benefits of nanotechnology have a large scope, increasing public and occupational exposure to nanomaterials have caused public concern regarding their adverse effects. 
Previous studies have shown that nanoparticles cause pulmonary injury, hepatotoxicity, renal toxicity, immunotoxicity, neurotoxicity and testis damage in animal models [5-11]. However, the female reproductive system is considerably more sensitive than other systems. Many studies regarding the toxicity of nanoparticles in the female reproductive system have been conducted $[12,13] . \mathrm{TiO}_{2}$ nanoparticles were found to cause ovarian dysfunction, and an intravenous injection of $\mathrm{SiO}_{2}$ and $\mathrm{TiO}_{2}$ into pregnant mice was observed to decrease the uterine weight and increase fetal reabsorption [14]. In addition, other nanoparticles, such as gold and carbon nanoparticles, can also penetrate the placental barrier in mice $[15,16]$. Some reports also demonstrated that nanoparticles can cross the mouse placental barrier and induce apoptosis in trophoblast cells $[17,18]$. A recent study founded that prenatal exposure to Cu-NPs caused a profound pulmonary inflammation in dams and strong immunomodulatory effects in offspring [19]. However, there are no studies describing the toxicity of Cu-NPs in the female reproductive system.

Copper is an essential trace element that helps maintain homeostatic function in living organisms [20]. Both copper deficiency and overload can lead to several diseases in humans [21]. In vivo and in vitro experiments have reported that copper is toxic to the reproductive system and a developing embryo $[22,23]$. Previous research showed that water-soluble copper salts exhibited the highest reproductive toxicity in worms followed by copper nanoparticles (Cu-NPs) and copper exposure in the soil23. The toxicity due $\mathrm{Cu}-\mathrm{NPs}$ exposure was probably caused by both the nanoparticles and $\mathrm{Cu}^{2+}$ released from the $\mathrm{Cu}-\mathrm{NPs}[24,25]$.

$\mathrm{Cu}-\mathrm{NPs}$ are now industrially produced and commercially available. They are extensively used as wood preservatives as well as an additive in lubricants, polymers/plastics, and metallic coating inks [26,27]. These nanoparticles are likely to be released into the environment and enter the human body via effluent, consumer products, or improper disposal [28]. Earlier studies have shown that $\mathrm{Cu}-\mathrm{NPs}$ can lead to injuries of the kidney, liver and spleen in mice as well as alter serum markers of the liver and kidney $[25,29]$. $\mathrm{Cu}-\mathrm{NPs}$ have also been shown to cause embryonic damage and change the physiology of zebrafish [10]. In addition, Cu-NPs decreased the reproductive capacity of red worms [24]. However, few studies have reported the toxicity of Cu-NPs in the reproductive systems of mammal.

In the present work, we prepared Cu-NPs (approximate $100 \mathrm{~nm}$ ) and examined their cytotoxicity in human extravillous trophoblast cells in vitro.
Furthermore, we explored the systemic reproductive toxicity of Cu-NPs in vivo in a mouse model by examining the sex hormones and evaluating the function and structure of the ovary, placenta and other associated reproductive organs. We discovered that $\mathrm{Cu}-\mathrm{NPs}$ exerted obvious reproductive toxicity on the mice by disrupting the balance of sex hormones as well as cytotoxicity on human extravillous trophoblast cells via ERK signaling and the mitochondria apoptosis pathway.

\section{Materials and Methods}

\section{Synthesis and characterization of Cu-NPs}

$\mathrm{Cu}-\mathrm{NPs}$ (approximate $100 \mathrm{~nm}$ ) were purchased from a nanomaterial company (Suzhou Tanfeng Graphene Tech. Inc. China) and had a mean nominal diameter of $100 \mathrm{~nm}$ and a purity of $99.5 \%$. All stock solutions were stored at $4^{\circ} \mathrm{C}$. Stock solutions of the nanoparticles $(200 \mu \mathrm{g} / \mathrm{mL})$ were prepared in D-5-W ( $5 \%$ dextrose in water) and diluted to the required concentrations using the cell culture medium. The nanoparticles were applied after $5 \mathrm{~min}$ of sonication and $1 \mathrm{~min}$ of vortexing. To explore the characteristics of the Cu-NPs synthesized in this experiment, transmission electron microscopy (TEM), X-ray diffraction (XRD), and fourier transform infrared spectrometer (FIRT) were all performed, and the diameter of the nanoparticles was estimated. X-ray photoelectron spectra (XPS) was performed using a Thermo Fisher ESCALAB250Xi instrument (Thermo Fisher, Waltham, MA, USA) with $\mathrm{Al}$ Ka radiation as the exciting source.

\section{Cell culture}

Cells were acquired from the Cell Bank of Typical Culture Collection (Chinese Academy of Sciences, Shanghai, China) and stored at our laboratory. The immortalized human first-trimester extravillous trophoblast cell line (HTR-8/SVneo) used in this study was grown in Dulbecco's modified Eagle's medium (DMEM) supplemented with 10\% (vol/vol) heat-inactivated fetal calf serum. The cells were maintained at $37^{\circ} \mathrm{C}$ in a humidified environment containing $5 \% \mathrm{CO}_{2}$.

\section{Cell viability survey}

To evaluate the cytotoxicity of Cu-NPs, HTR-8/SVneo cells in the logarithmic growth phase were seeded into a 96-well culture plate at 2000 cells per well and incubated at $37{ }^{\circ} \mathrm{C}$ in a humidified environment containing $\mathrm{CO}_{2}$ for $24 \mathrm{~h}$ until the cells adhered to the plate. Then, the cells were treated with various concentrations of $\mathrm{Cu}-\mathrm{NPs}(0,5,10,20$, or 40 $\mu \mathrm{g} / \mathrm{mL}$ ) for both $48 \mathrm{~h}$ and $72 \mathrm{~h}$, after which cell viability was assessed using a CCK-8 detection kit 
(Sigma, Milwaukee, WI, USA) according to the manufacturer's instructions. Briefly, after treatment with the different concentrations of Cu-NPs, $20 \mu \mathrm{l}$ of CCK-8 solution was added to each well, and the plate was incubated at $37^{\circ} \mathrm{C}$ for $2 \mathrm{~h}$. Then, the inhibition of HTR-8/SVneo cell proliferation was determined based on the absorbance measurements at a wavelength of $450 \mathrm{~nm}$ using a microplate reader (Bio-Rad).

\section{Apoptosis survey}

HTR-8/SVneo cells were seeded into six-well plates $\left(1 \times 10^{6}\right.$ per well). After the cells were cultured for $24 \mathrm{~h}$, the medium was replaced with fresh complete medium, and the cells were treated with 0 , $5,10,20$, or $40 \mu \mathrm{g} / \mathrm{mL} \mathrm{Cu}-\mathrm{NPs}$ for either $48 \mathrm{~h}$ or $72 \mathrm{~h}$. To measure the extent of apoptosis, the cells were then harvested and stained with propidium iodide (PI) and annexin V-FITC using a commercially available kit (BD Pharmingen, San Diego, CA, USA) according to the manufacturer's instructions and were analyzed by flow cytometry on a MACSQuant Analyzer (Miltenyi Biotec). The percentage of apoptotic cells was calculated using FlowJo 7.6.

\section{Determination of cell cycle}

Cell cycle analysis was conducted by PI staining followed by flow cytometry. Briefly, HTR-8/SVneo cells were treated with different concentrations of nanoparticles $(5,10,20$, and $40 \mu \mathrm{g} / \mathrm{mL})$ for $48 \mathrm{~h}$ or72 $\mathrm{h}$. Treated and untreated control cells were harvested, washed twice with phosphate-buffered saline (PBS), and fixed in ice-cold ethanol $(70 \%)$ for $4 \mathrm{~h}$ at $4^{\circ} \mathrm{C}$. The fixed cells were washed, pelleted, resuspended in PBS, stained with a solution containing PI and RNase $(40 \mu \mathrm{g} / \mathrm{mL}$ and $100 \mu \mathrm{g} / \mathrm{mL}$, respectively) and incubated at $37^{\circ} \mathrm{C}$ for $30 \mathrm{~min}$ until analysis. Flow cytometry was performed using an Epics Altra flow cytometer (Beckman Coulter) at an excitation wavelength of $488 \mathrm{~nm}$ and an emission wavelength of $610 \mathrm{~nm}$. The data collected for $2 \times 10^{4}$ cells per conditionwereanalyzed using WinMDI 2.8 software.

\section{Measurement of the mitochondrial membrane potential}

HTR-8/SVneo cells in six-well plates were treated with $\mathrm{Cu}-\mathrm{NPs}(10,20$, or $40 \mathrm{mg} / \mathrm{ml})$ for $12 \mathrm{~h}$. The mitochondrial membrane potential (MMP) was monitored using the JC-1 mitochondrial membrane potential detection kit (Biotium, Hayward, CA, USA). In healthy cells, JC-1 (a fluorescent cationic dye) enters the negatively charged mitochondria, where the dye aggregates and fluoresces red. In cells in which the MMP has collapsed, JC-1 exists as monomers throughout the cell, which fluoresce green.
Consequently, mitochondrial depolarization is indicated by a decrease in the ratio of the red:green fluorescence intensities. Data acquisition was performed using a MACSQuant Analyzer BD-FACS Canto II (Miltenyi Biotec, Becton-Dickinson, San Jose, CA, USA), and the data were analyzed using FlowJo 7.6 software (Tree Star, Inc., Ashland, MA, USA).

\section{Western blotting analysis}

Briefly, HTR-8/SVneo cells were treated with $\mathrm{Cu}-\mathrm{NPs}$ at a concentration of $5 \mu \mathrm{g} / \mathrm{ml}, 10 \mu \mathrm{g} / \mathrm{ml}$, or $20 \mu \mathrm{g} / \mathrm{ml}$. After the cells were treated for $24 \mathrm{~h}$, they were pelleted by centrifugation and washed twice with ice-cold PBS. The resultant cell pellets were resuspended in $1 \mathrm{ml}$ of cytosol extraction buffer containing DTT and protease inhibitor and incubated for $15 \mathrm{~min}$ on ice. After homogenization, unbroken cells and large debris were removed by centrifugation. The cell lysates were loaded on a $10 \%$ gel and subjected to SDS-PAGE followed by Western blotting with anti-RAS, anti-BRAF, anti-ERK, and anti-MITF antibodies (CST, Danvers, MA, USA). Bound antibody was detected with a peroxidase-conjugated anti-rabbit antibody followed by chemiluminescence (ECL System, Waltham, MA, USA) and exposure via autoradiography. The same blots were then probed for $\beta$-actin (CST).

\section{In vivo reproductive and embryonic toxicity assays}

To explore the reproductive and embryonic toxicity in female mice and their off spring, 24 Non-pregnant ICR female mice were randomly divided into four groups (six per group), comprising two control (pregnant and nongravid) groups and two Cu-NP-treated groups (pregnant and nongravid). Pregnant groups with 12 males and 12 females mice were randomly divided into two of four groups, then female and male mice were mated at the ratio of 1:1. After mating, vaginal smear examinations were performed on female animals the following morning. The day on which either sperm or a vaginal plug was detected was regarded as gravidity day 0 (GD0). Pregnant animals were randomly allocated into one of two treatment groups. Mice in the Cu-NPs treatment group were injected with $\mathrm{Cu}-\mathrm{NPs}$ for 14 days,via tail vein at the dosage of $100 \mu \mathrm{g}$ per day per mice, and the control treatment mice were injected with $5 \%$ glucose. The pregnants were treated with $\mathrm{Cu}-\mathrm{NPs}$ begin 7 days before mating continued to GD7. At GD14, the pregnant female sunders went Caesarean section and were then euthanized. Recorded pregnancy outcomes included the numbers of corpora lutea, implantation sites, live fetuses, dead fetuses and resorbed fetuses, as well as fetal gender (Sigma). Blood, ovarian, 
uterine, and placental samples were also collected. Because the hypothalamic-pituitary-testicular axis is important for reproductive and embryonic development, we tested the serum levels of associated hormones and growth factors, including estrogen, follicle-stimulating hormone (FSH), and progesterone (Sigma). The placenta and ovaries from the control-and $\mathrm{Cu}-\mathrm{NP}$-treated groups were all harvested and fixed with $4 \%$ paraformaldehyde for at least $24 \mathrm{~h}$. Then, the organs were embedded in paraffin, and hematoxylin and eosin (H\&E) staining was performed to observe changes in the organ structure. ICR mice aged 6-8 weeks old and weighing approximately 25-30 g were fed a standard pellet diet supplemented with vitamins and provided water. The mice were housed in cages at the departmental animal facility with a $12 \mathrm{~h}$ light/dark cycle at a standard temperature. The animals were acclimatized for one week prior to initiation of the experiments and did not show any pathological symptoms.

All the animal experiments were performed in accordance with the Guide for the Care and Use of Laboratory Animals and were approved by the Bioethics Committee of Second Military Medical University, and all the experiments were performed following relevant guidelines and regulations of Second Military Medical University.

\section{Data and statistical analyses}

The data are presented as the means \pm S.D. Paired Student's t-tests and ANOVA were used to analyze differences in the hormone levels, liver and kidney function indexes, and mitochondrial potential. Significance was established at $P<0.05$.

\section{Results}

\section{Characterization of Cu-NPs}

The Cu-NPs were first characterized by TEM. The acquired images showed that the $\mathrm{Cu}-\mathrm{NPs}$ were all spherical and homogeneous (Figure 1A). The diameter of the $\mathrm{Cu}-\mathrm{NPs}$ in suspension was approximately $100 \mathrm{~nm}$, and the shape and density of the nanoparticles was round and homogeneous, respectively. Further analysis of the diameter (Figure 1B) indicated that $\mathrm{Cu}-\mathrm{NPs}$ had a diameter between approximately 50 and $150 \mathrm{~nm}$.

Figure 1C presents the FTIR spectra of the Cu-NPs, showing their composition and quality. The band at $616 \mathrm{~cm}^{-1}$ correlates with the stretching vibrations of copper monomer, and there was no significant vibrations of other materials and oxidation state of cooper.

Figure 1D of XRD showed the main diffraction pattern and interplanar spacing of the $\mathrm{Cu}-\mathrm{NPs}$ were similar to those in the standard diffraction pattern of copper monomer. And, some weak peaks of cuprous oxide in the XRD were also tested. Furthermore, no strong characteristic peaks of cooper oxide were detected, suggesting the high purity of the Cu-NPs.

XPS was used to further study the element composition and chemical state of Cu-NPs. Cu2p scan of $\mathrm{Cu}-\mathrm{NPs}$ showed that the peak was located at 932.5 $\mathrm{eV}$, which is consistent with the cooper in zero and one valance (Figure 1E). As we know, the standard peak of cooper in zero and one valance is overlap, which make it difficult to certain the valance of cooper just by the XPS scan. Furthermore, the standard peak of $\mathrm{Cu}$ (II) is located between $933.5 \mathrm{eV}$ to $935 \mathrm{eV}$. In the XPS scan, the peak of C1s was calibrated to $284.8 \mathrm{eV}$.

Based on the different methods to explore the characterization, the main valence state of $\mathrm{Cu}-\mathrm{NPs}$ was zero, the cooper (I) may also have little content, and there was no cooper in two valence. So, the $\mathrm{Cu}-\mathrm{NPs}$ prepared for our experiments were of good quality and purity without any surface modification, and can be used for further biological research.

\section{Cu-NPs inhibit proliferation and induce apoptosis of HTR-8/SVneo cells in vitro}

After HTR-8/SVneo cells were treated with different concentrations of $\mathrm{Cu}-\mathrm{NPs}$, the results showed that the proliferation of HTR-8/SVneo cells was significantly inhibited, and the inhibition ratios of cells at $48 \mathrm{~h}$ and $72 \mathrm{~h}$ were nearly $80 \%$ and $90 \%$, respectively, at the concentration of $40 \mu \mathrm{g} / \mathrm{ml}$; these differences were statistically significant when compared to the corresponding values in the control group (Figure 2B).

To evaluate the apoptotic effect of Cu-NPs on HTR-8/SVneo cells, the cells were exposed to 5, 10, 20, and $40 \mu \mathrm{g} / \mathrm{ml} \mathrm{Cu}-\mathrm{NPs}$ for $48 \mathrm{~h}$ or $72 \mathrm{~h}$. As shown in Figure 2A, Cu-NPs induced apoptosis in HTR-8/ SVneo cells in a time-and concentration-dependent manner; furthermore, whereas approximately $20 \%$ of cells were apoptotic after exposure to $40 \mu \mathrm{g} / \mathrm{ml}$ Cu-NPs for $48 \mathrm{~h}$, approximately $60 \%$ of cells were apoptotic after exposure to the same concentration for $72 \mathrm{~h}$. In contrast, the control group exhibited $7.97 \%$ apoptosis rate.

\section{Cu-NPs induced cell cycle arrest in HTR-8/SVneo cells}

The early effects of DNA damage can be observed during cell cycle progression. Cells with damaged DNA will accumulate in the G1, S, or G2/M phases. A cell cycle progression analysis was performed and showed that HTR-8/SVneo cells were highly arrested by Cu-NPs in a dose-and time-dependent manner. Approximately $38 \%$ of cells exposed to $40 \mu \mathrm{g} / \mathrm{mL} \mathrm{Cu}-\mathrm{NPs}$ for $48 \mathrm{~h}$ were arrested 
at the G2/M phase, whereas $83 \%$ of cells exposed to $40 \mu \mathrm{g} / \mathrm{mL}$ of $\mathrm{Cu}-\mathrm{NPs}$ for $72 \mathrm{~h}$ were arrested at the $\mathrm{G} 2 / \mathrm{M}$ phase. At the same time, $\mathrm{S}$ phase cells became a little higher than the control group at the concentration of $40 \mu \mathrm{g} / \mathrm{mL}$ of $48 \mathrm{~h}$, however, this phenomenon was observed at the $72 \mathrm{~h}$, which indicated that Cu-NPs can induce the G2/M and S phase arrest and the $S$ phase arrest cells were induced apoptosis more easily than the G2/M arrest cells. Additionally, Cu-NP-treated cells exhibited a decrease in the G1 population accompanied by an increase in the G2/M population. The percentage of cells in each phase of the cell cycle was compared to that of controls (Figure 2C, D).

A

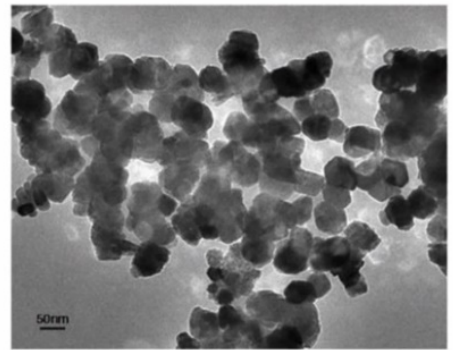

C

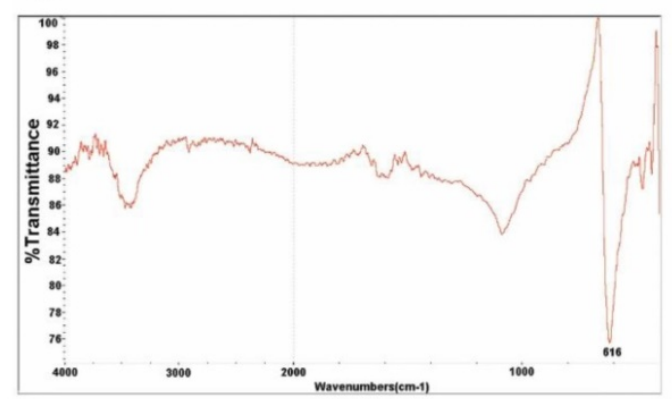

E

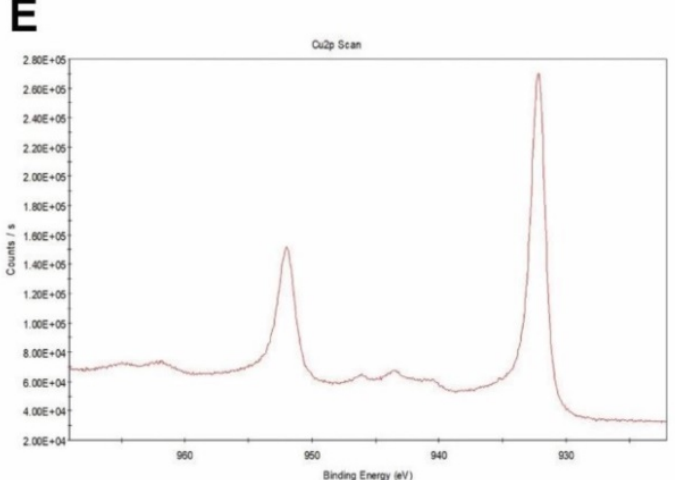

\section{Reproductive, embryonic, renal and liver toxicity of $\mathrm{Cu}-\mathrm{NPs}$ in vivo}

To explore the reproductive toxicity of $\mathrm{Cu}-\mathrm{NPs}$, we treated pregnant mice with either $\mathrm{Cu}-\mathrm{NPs}$ at a dosage $100 \mu g$ per day per mice or with $5 \%$ glucose at the same volume. None of the pregnant mice treated with $\mathrm{Cu}-\mathrm{NPs}$ experienced spontaneous abortion, and no died embryos were present. During our research, we recoded the mice weight of different group, and we found that there was no change in the $\mathrm{Cu}-\mathrm{NPs}$ treated mice, including both pregnant and unpregnant group (Figure S1).

The H\&E staining results showed that there was inflammatory cell infiltration as well as congestion and edema in the placentas of $\mathrm{Cu}-\mathrm{NP}$-treated pregnant mice (Figure 3A).

B

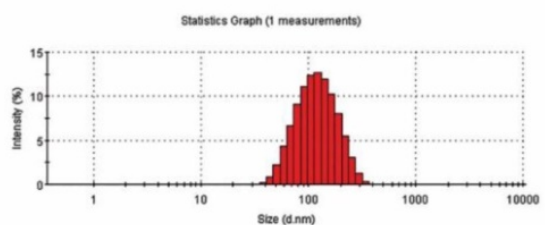

D

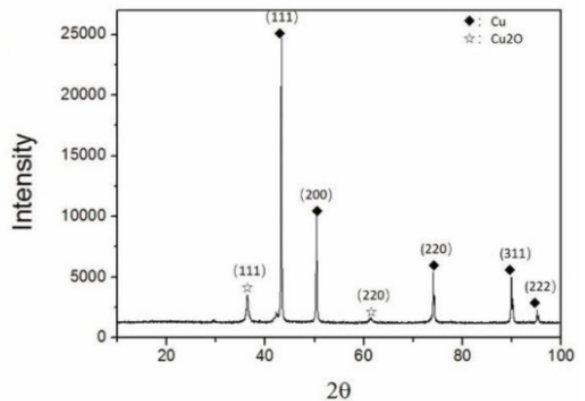

Figure 1. Characterization of Cu-NPs. (A) TEM of Cu-NPs showing that the Cu-NPs were spherical and homogeneous. (B) Diameter assessment of Cu-NPs indicating that the diameter of Cu-NPs ranged between approximately 50 and $150 \mathrm{~nm}$. (C) FIRT assay. (D) XRD patterns assay. ( $\bullet$ : Peak of cooper in zero valence, ț: Peak of cuprous(I) oxide) (E) XPS scan assay. 
A

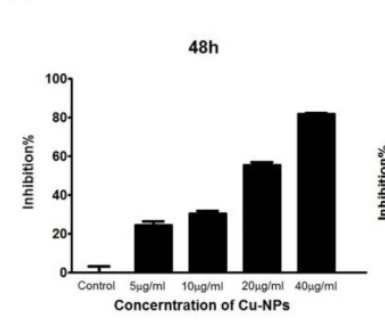

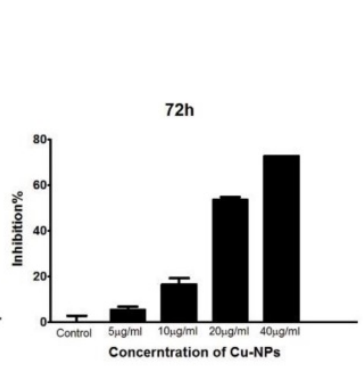

B

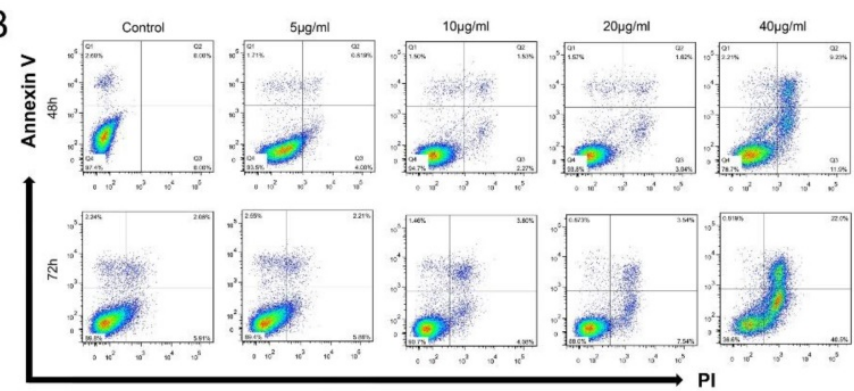

C
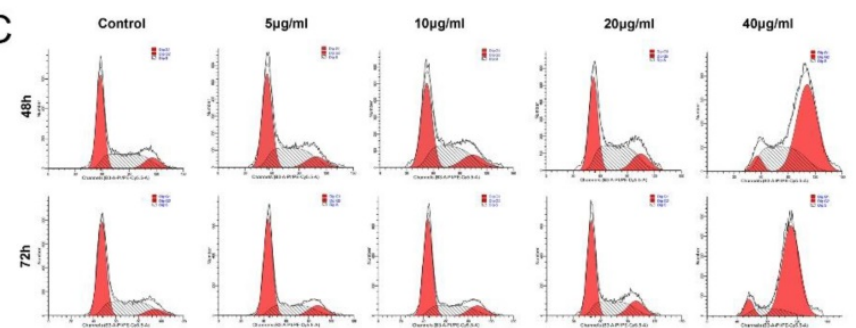

D
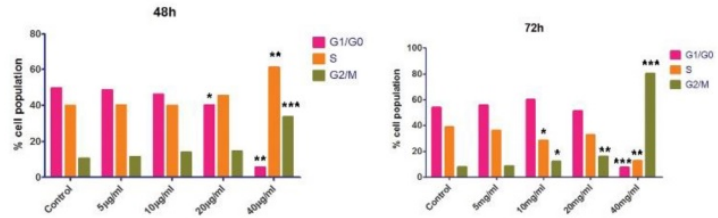

Figure 2. Cytotoxicity of Cu-NPs inHTR-8/SVneo cells. (A) The inhibitory effects of Cu-NPs on proliferation as determined by theCCK8 assay. Cu-NPs can strongly inhibit the proliferation of HTR-8/SVneo cells, and the IC50values after48 $\mathrm{h}$ and $72 \mathrm{hof}$ treatment were $20 \mu \mathrm{g} / \mathrm{ml}$ and $10 \mu g / \mathrm{ml}, \mathrm{respectively.} \mathrm{(B)} \mathrm{Flow}$ cytometry analysis of HTR-8/SVneo cells treated with different concentrations of Cu-NPs for $48 \mathrm{~h}$ and $72 \mathrm{~h}$ followed by staining with annexin V-FITC and PI. Significant increases in apoptosis were observed in HTR-8/SVneo cells in a dose- and time-dependent manner. The upper-left quadrant in each panel indicates the necrotic population, the upper-right quadrant shows the late apoptotic population, and the lower right quadrant shows the early apoptotic population. (C) Cell cycle progression assay of HTR-8/SVneo cells treated with different concentrations of Cu-NPs. Cu-NPs can cause significantly cell cycle arrest at G2/M in a dose-dependent manner, with the most profound results observed at a highest concentration of $40 \mu \mathrm{g} / \mathrm{ml}$. (D) Statistics of the cell cycle progression of Cu-NP-treated HTR-8/SVneo cells (Student't test, $* P<0.05, * * p<0.01, * * * P<0.01$ ).

A
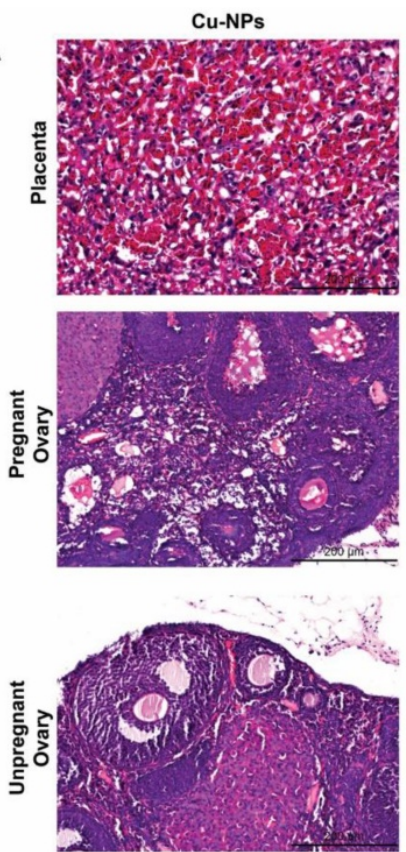

Control
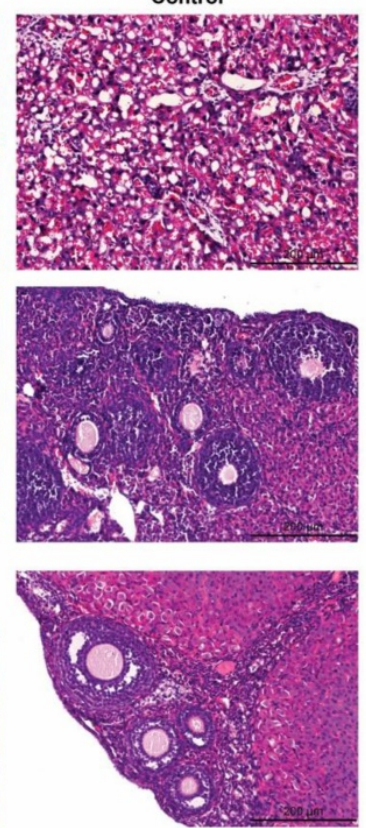

B

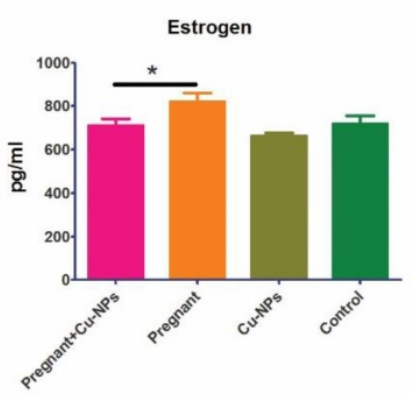

D

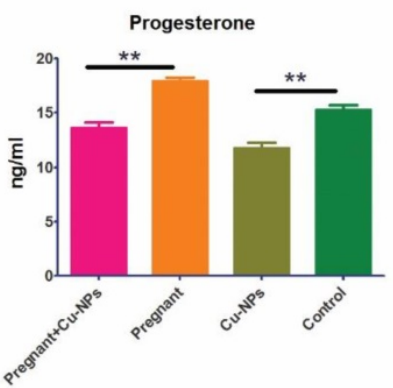

C

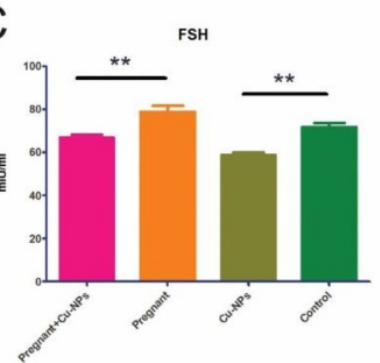

$\mathrm{E}$

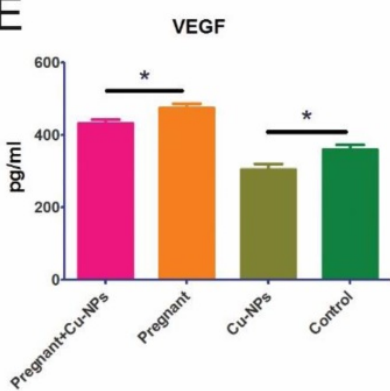

Figure 3. Reproductive toxicity of Cu-NPs. (A) H\&E-stained images of the placentas and ovaries from Cu-NPs-treated and control-treated mice. More inflammatory cells significantly infiltrated the placentas of Cu-NP-treated mice, and the number of primordial follicles and corpus lutea was significantly decreased. (B) Measurement of serum estrogen levels. The serum estrogen levels in the Cu-NPs-treated pregnant mice were lower than those in untreated pregnant mice. There was no statistically significant difference between the treated and untreated nongravid mice ( $* P<0.05$, Student's t-test). (C) Measurement of serum FSH levels. The serum FSH levels in the Cu-NP-treated mice were lower than those in untreated mice in both the pregnant group and nongravid group (**P<0.01, Student's t-test). (D) Measurement of serum progesterone levels. The serum progesterone levels in the Cu-NP-treated mice were lower than those in untreated mice in both the pregnant group and nongravid group ( $* * P<0.01$, Student's t-test). (E) Measurement of serum VEGF levels. The serum VEGF levels in the Cu-NP-treated mice were lower than those in untreated mice in both the pregnant group and nongravid group $\left({ }^{*} P<0.05\right.$, Student's t-test). 
Measurements of the serum levels of sex hormones and growth factors showed that $\mathrm{Cu}-\mathrm{NPs}$ can significantly decrease FSH, progesterone, and vascular endothelial growth factor (VEGF) levels in both pregnant and nongravid mice. The estrogen levels in the pregnant group were inhibited by $\mathrm{Cu}-\mathrm{NPs}$; however, the difference of nongravid group was not as obvious (Figure 3B, C, D).

Furthermore, we tested renal and liver function serum markers to determine renal and liver toxicity, respectively. The renal function markers did not indicate renal toxicity due to $\mathrm{Cu}-\mathrm{NPs}$, as there were lower levels of BUN, UA and CR in the Cu-NPs- treated group (Figure 4A, B). The serum ALB levels in $\mathrm{Cu}-\mathrm{NPs}$-treated pregnant and nongravid mice were slightly lower than those in the corresponding groups treated with vehicle (Figure 4C). Other serum liver function markers such as ALP, and GLB in the $\mathrm{Cu}-\mathrm{NP}$-treated groups were not lower than those in the untreated groups (Figure 4D, E, F). We also performed H\&E staining of the kidney, liver, lung, and uterus from $\mathrm{Cu}-\mathrm{NPs}$ treated mice and observed no significant changes, which suggested that $\mathrm{Cu}-\mathrm{NPs}$ did not exert obvious renal and liver toxicity at this concentration (Figure 4G).
A

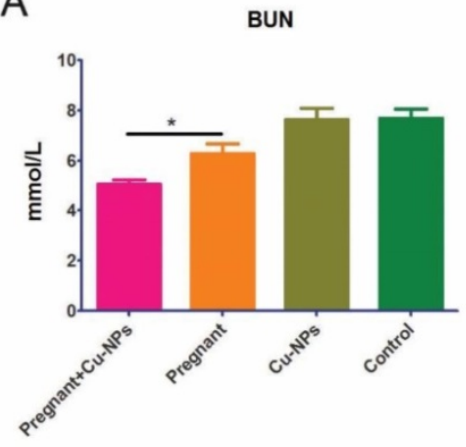

C

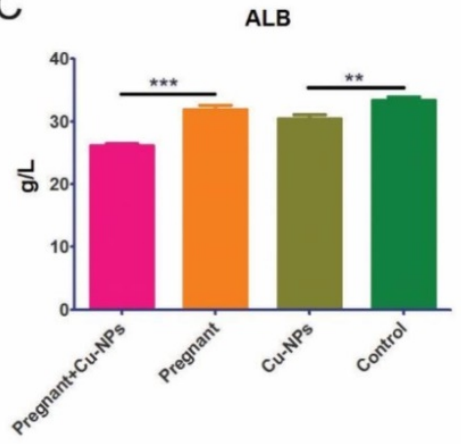

$\mathrm{E}$

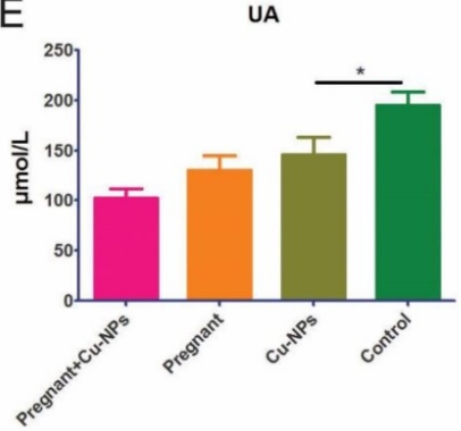

B
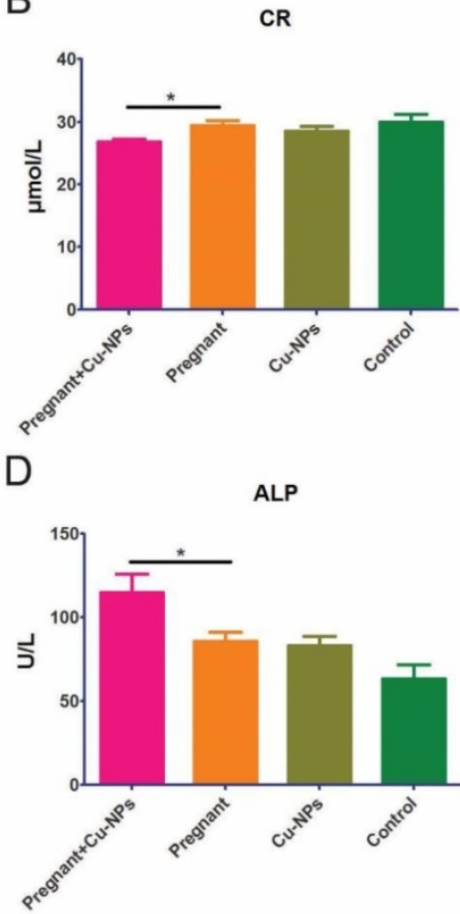

$\mathrm{F}$

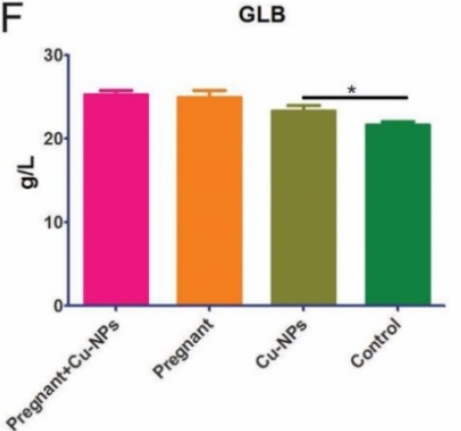

G

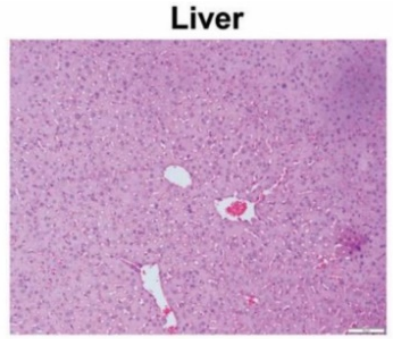

Kidney

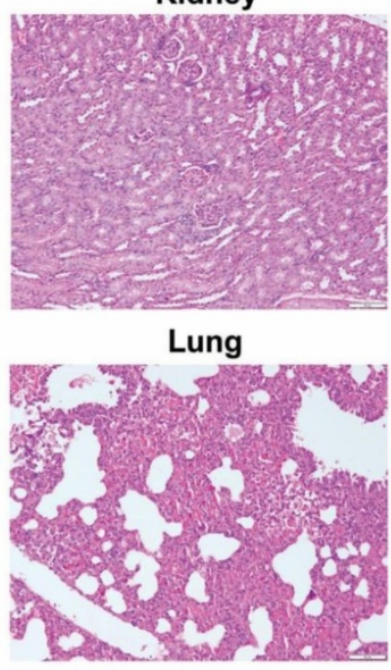

Uterus

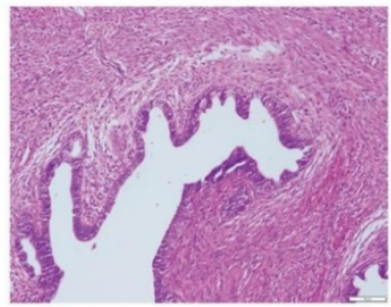

Figure 4. Cu-NP renal and liver toxicity. (A) Measurement of serum BUN levels. The serum BUN levels in the Cu-NP-treated mice were lower than those in the untreated pregnant mice. There was no statistically significant difference between the treated and untreated nongravid mice $(* P<0.05$, Student's t-test). (B) Measurement of serum CR levels. The serum CR levels in the Cu-NP-treated mice were lower than those in the untreated pregnant mice. There was no statistically significant difference between the treated and untreated nongravid mice ( ${ }^{*} P<0.05$, Student's t-test). (C) Measurement of serum ALB levels. The serum ALB levels in the Cu-NP-treated mice were lower than those in the untreated mice in both the pregnant group and nongravid group ( $* * P<0.01$, $* * * P<0.001$, Student's t-test). (D) Measurement of serum ALP levels. The serum ALP levels in the Cu-NP-treated mice were higher than those in the untreated pregnant mice. There was no statistically significant difference between treated and untreated nongravid mice $(* P<0.05$, Student's t-test). (E) Measurement of serum UA levels. The serum UA levels in the Cu-NP-treated mice were lower than those in the untreated nongravid mice. There was no statistically significant difference between treated and untreated pregnant mice $(* P<0.05$, Student's t-test). (F) Measurement of serum GLB levels. The serum GLB levels in the Cu-NP-treated mice were higher than those in the untreated nongravid mice. There was no statistically significant difference between treated and untreated pregnant mice $(* P<0.05$, Student's t-test). (G) $H \& E$-stained images of the kidney, liver, lung, and uterus showing that Cu-NPs at a total dose of $4 \mathrm{mg} / \mathrm{kg}$ per day did not cause any significant histological changes. 


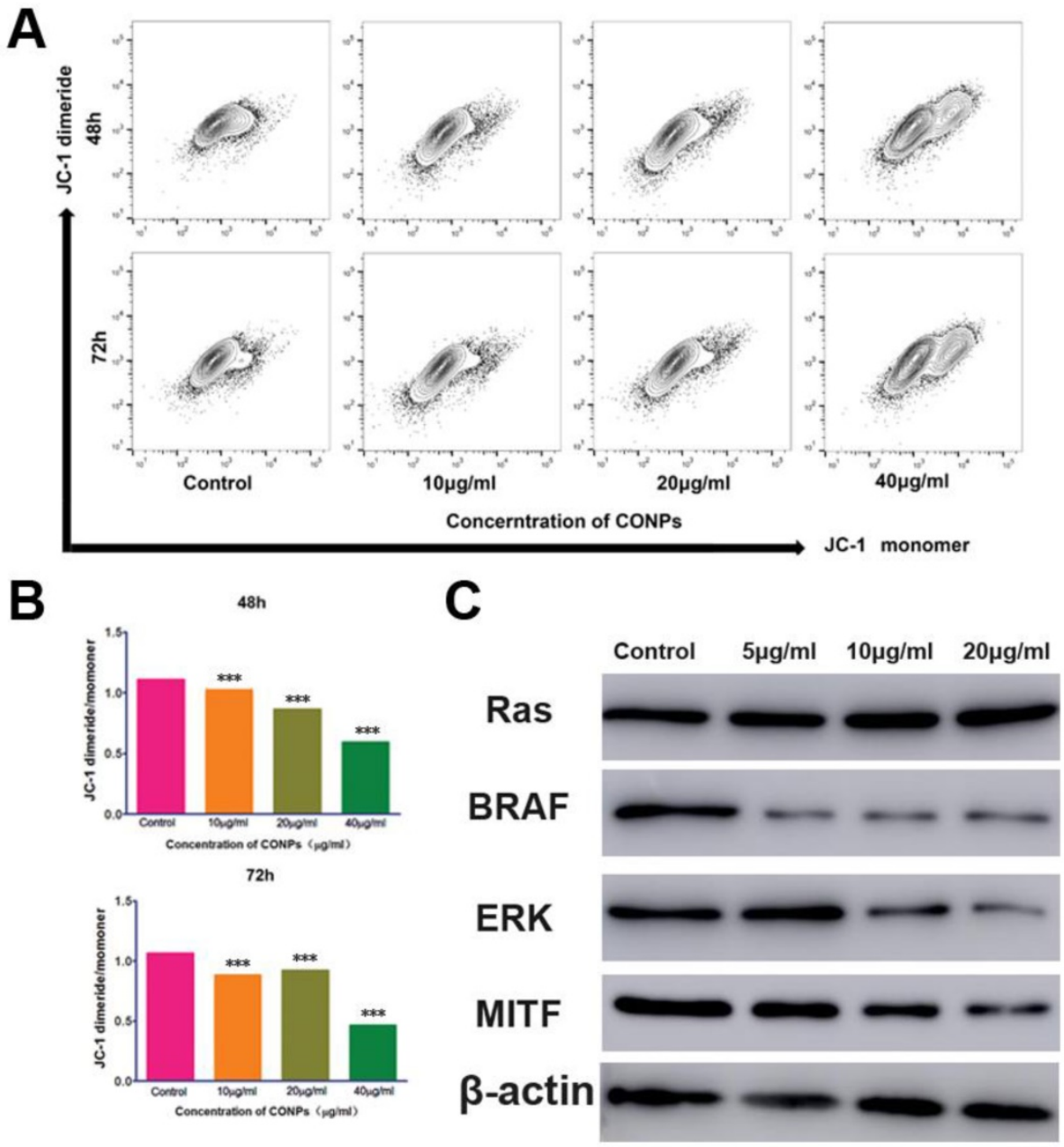

Figure 5. Measurement of the mitochondrial membrane potential and the expression of proteins in the ERK signaling pathway. (A) Flow cytometry showed that the mitochondrialmembrane potential of HTR-8/SVneo cells significantly decreased after treatment with Cu-NPs for $48 \mathrm{~h}$ and $72 \mathrm{~h}$. (B) Fluorescence intensity ratio of JC-1 dimers to JC-1 monomer (***P<0.01). (C) Western blot assay of proteins in the ERK signaling pathway. The BRAF, ERK, and MITF protein expression levels were significantly decreased after Cu-NPs treatment for 48 h. The expression of RAS did not show obvious changes.

\section{Cu-NPs can block the ERK signaling pathway and decrease the mitochondrial membrane potential}

As Cu-NPs exhibited significant reproductive toxicity and have been shown to induce the apoptosis of human villous trophoblast cells, it is necessary to explore the mechanism. First, we found that $\mathrm{Cu}-\mathrm{NPs}$ can significantly inhibit the MMP of human villous trophoblast cells (Figure 5A, B), which suggests that $\mathrm{Cu}-\mathrm{NPs}$ can activate the mitochondria-mediated apoptosis signaling pathway. As ERK signaling is also important for the proliferation of various cell types, we also tested proteins in the ERK signaling pathway. The results showed that $\mathrm{Cu}-\mathrm{NPs}$ can significantly decrease the expression of BRAF, ERK, and MITF, the latter of which is an important target gene of ERK. However, Cu-NPs did not influence the expression of RAS (Figure 5C).

\section{Discussion}

Because of their specific properties, copper nanomaterials are widely used in industrial products, consumer products, drug formulations and devices; some examples including solar cells, lithium-ion batteries, lubricant oil polymers/plastics, ink/ceramic gas sensors and electronics. They exist naturally in the environment and can be produced or engineered. In the environment, these particles can be taken up by organisms. Toxicologists and scholars have recognized the potential health risks from nanomaterials, and previous research has demonstrated nano-copper toxicity with in the soil plant system and ecosystem $^{24,30}$. In some studies, copper nanoparticles have been found be toxic to mice at an LD50 of $413 \mathrm{mg} / \mathrm{kg}$ via oral gavage; this dose led to pathological changes and injuries to the kidney, liver, and spleen of mice as well as induced hepatic and renal dysfunction ${ }^{29}$. Adamcakova-Dodd A,et al [19] founded that prenatal 
inhalation exposure to copper nanoparticles did not identify histopathology of placentas but caused a profound pulmonary inflammation in dams and strong immunomodulatory effects in offspring. To explore the reproductive toxicity of $\mathrm{Cu}-\mathrm{NPs}$ without potential confounding factors, followed our preliminary experiments and treatment with 10 $\mathrm{mg} / \mathrm{kg} \mathrm{TiO}_{2} \mathrm{NPs}$ via intragastric administration resulted in the most severe organ damage for the mice.in the present study were exposed to Cu-NPs at a dosage $100 \mu \mathrm{g}$ per day per mice(approximately $4 \mathrm{mg} / \mathrm{kg}$ ) via tail vein injection, and we did not observe significant histological changes in the kidney, liver, lung, and uterus from Cu-NP-treated mice compared to those from control-treated mice. The serum levels of renal function markers (BUN, UA and CR) and liver function markers (ALP and GLB) did not show obvious differences between the $\mathrm{Cu}-\mathrm{NP}$-treated groups and the control-treated group. These results suggested that $\mathrm{Cu}-\mathrm{NPs}$ at the concentration administered did not exert obvious renal and liver toxicity (Figure 4G). Different administration methods of $\mathrm{Cu}-\mathrm{NPs}$ have different effects on the liver and kidneys in mice. A previous study [31,32] showed that the toxicity of Cu-NPs was probably due to both the nanoparticles themselves and released $\mathrm{Cu}^{2+}$. When $\mathrm{Cu}-\mathrm{NPs}$ enter the gastric lumen, they rapidly react with the hydrogen ions of the gastric juice and convert into their ionic states. Thus, both copper iron overload and metabolic alkalosis contribute to the toxicity of Cu-NPs.

Furthermore, nanoparticles may adversely impact the reproductive system and fertility in mice $[33,34]$. Early studies revealed that titanium dioxide nanoparticles can enter ovarian granulosa cells and inhibit the synthesis of estradiol [35]. Triptolideloaded nanoparticles caused impaired ovarian structure and function, subfertility, and increased follicular apoptosis [36,37]. In our experiment, we found inflammatory cell infiltration as well as congestion and edema in the placenta and ovaries of $\mathrm{Cu}-\mathrm{NPs}$-treated mice (Figure 3A). The number of primordial follicles and corpus lutea was significantly decreased compared to control-treated animals. $\mathrm{Cu}$-NPs exposure to primordial follicles can significantly decrease the FSH, progesterone, estrogen, and VEGF levels in both pregnant and nongravid mice. A shortened early-follicular phase and down-regulation of estrogen and progesterone result in a diminished ovarian reserve [38]. Maintenance of the progression of primordial follicles into developing follicles is crucial for ovarian function throughout a woman's reproductive life.

Normal placental development is required for embryonic growth, and placental dysfunction has been associated with miscarriage and fetal growth restriction [39]. In addition, normal placental development requires the coordinated expression of VEGF and its receptor, soluble fms-like tyrosine kinase-1 (sFLt-1) [40]. The placentas of mice treated with nSP70showed variable abnormalities compared to those from control mice. The plasma levels of sFLt-1 in nSP70- and nano- $\mathrm{TiO}_{2}$-treated mice were significantly lower than those in control mice [14]. Our results were consistent with previous studies, as the data indicated that $\mathrm{Cu}-\mathrm{NPs}$ can cause pathological change in the placenta. VEGF was also significantly inhibited in both pregnant and nongravid mice.

Our in vivo experiments demonstrated that Cu-NPs may adversely impact the reproductive system and fertility of mice. However, there are no reports regarding the toxicity of $\mathrm{Cu}-\mathrm{NPs}$ on the human reproductive system and fertility. To further investigate the potential reproductive toxicity of Cu-NPs in humans, multiple assays were conducted to analyze whether $\mathrm{Cu}-\mathrm{NPs}$ can reduce the viability of human extravillous trophoblastic cells.

During early pregnancy, extra villous trophoblasts invade and migrate to affect spiral artery remodeling. Failed or incomplete spiral artery remodeling is associated with various fertility disorders, including miscarriage, intrauterine growth restriction (IUGR) and placental abruption [41].

First, to investigate the inhibitory effect of Cu-NPs on proliferation, the CCK-8 assay was used to measure cell viability after nanoparticle treatment. As shown in Figure 2B, the proliferation of the trophoblastic cell lines was decreased in a time- and concentration-dependent manner. Based on the CCK-8 results, we performed flow cytometry to determine the apoptotic population among the Cu-NP-treated trophoblastic cell lines. Figure 2A shows that $\mathrm{Cu}-\mathrm{NPs}$ induced apoptosis in HTR-8/ SVneo cells in a time- and concentration-dependent manner. Some research has demonstrated that different nanoparticles can suppress cell viability by different mechanisms. Some types of nanoparticles can induce apoptosis in cells whereas others can activate cell necrosis [8,42]. To investigate the mechanism of the cytotoxicity of $\mathrm{Cu}-\mathrm{NPs}$, we performed flow cytometry to analyze the effects of $\mathrm{Cu}-\mathrm{NPs}$ on cell death and the cell cycle. Our investigation revealed that $\mathrm{Cu}-\mathrm{NPs}$ suppressed the proliferation of HTR-8/SVneo cells and caused cell cycle arrest at G2/M phase as shown in Figure 2C.

Mitochondria have key roles during the early stages of apoptosis. Loss of the MMP is a crucial step in triggering apoptosis [43]. To determine the effect of $\mathrm{Cu}-\mathrm{NPs}$ on mitochondrial function as well as their role in inducing cell death, the JC-1 assay was used to 
analyze the MMP as described earlier. We measured the loss of the MMP in cells treated with Cu-NPs, as shown in Figure 5. Ag-NPs and other inorganic nanomaterials can also cause the loss of the MMP and activate the mitochondria-mediated apoptosis signaling pathway [44]

As we know that ERK signaling pathway is sensitive to the cooper concentration and Cu-NPs is made up with cooper element, it is necessary to explore the effects of $\mathrm{Cu}-\mathrm{NPs}$ on the ERK signaling pathway. Recent studies discovered that activating the ERK pathway can also contribute to inhibited proliferation and the induction of apoptosis [45,46]. $\mathrm{Cu}-\mathrm{NPs}$ are toxic to the skin, and dermal factors and ERK may be key regulators of this cytotoxicity [47]. The epidermal growth factor receptor (EGFR) pathway leads to numerous effects such as anti-apoptosis and cell cycle arrest [48]. Manna [25] found that a moderate concentration of $\mathrm{Cu}-\mathrm{NPs}$ inhibited activation of the EGFR pathway. Downregulation of the RAS/RAF/MEK/ERK signaling pathway was closely associated with hepatic cell apoptosis and proliferation. The results of our investigation are consistent with previous studies. Other nanomaterials may indirectly cause DNA damage by promoting oxidative stress and inflammatory responses via dysfunction of the mitochondria or inflammasomes [12]. In our study, inflammatory cytokines were not investigated owing to current limitations, and future studies may be required.

\section{Conclusions}

The results of our study suggest that $\mathrm{Cu}-\mathrm{NPs}$ induced ovarian and placental pathophysiology and dysfunction in mice. Cu-NPs also induced apoptosis and suppressed proliferation in human HTR-8/SVneo cells in a time- and dose-dependent manner and arrested the cell cycle in a time-dependent manner And, the ERK signaling pathway and mitochondrion disfunction all made great contribution to the toxicity of $\mathrm{Cu}-\mathrm{NPs}$. To the best of our knowledge, this is the first report of the cytotoxicity of nanocopper in human extravillous trophoblast cells; furthermore, our study provides information about the safety and care that should be taken before using copper particles in nanomaterials.

\section{Supplementary Material}

Supplementary figures and tables. http://www.ijbs.com/v14p1834s1.pdf

\section{Acknowledgements}

The authors would like to thank Xin-Gang Liu, Xiao-Di Yang, Yan-Jiao Zhao (Department of Public
Technique Platform, Laboratory of Advanced Material, Fu Dan University) for their useful discussion and technical assistance with the sample analysis. The present study was financed by the National Natural Science Foundation of China (Grant Number: 81472771, 31271250).

\section{Author Contributions}

Conceived and designed the experiments: $\mathrm{ZCH}$, WY, SQQ and GH. Performed the experiments: $\mathrm{ZCH}$, WY, SQQ, HJJ, CK, Analyzed the data: WY Contributed reagents/materials/analysis tools: $\mathrm{ZCH}$, XLL, WX, FXX. Wrote the paper: ZCH, GH.

\section{Competing Interests}

The authors have declared that no competing interest exists.

\section{References}

1. Augustin MA, Sanguansri P. Nanostructured materials in the food industry. Adv Food Nutr Res. 2009; 5:183-213.

2. Bowman DM, vanCalster G, Friedrichs S. Nanomaterials and regulation of cosmetics. Nat Nanotechnol. 2010; 5:92.

3. Petros RA, DeSimone JM. Strategies in the design of nanoparticles fortherapeutic applications. Nat Rev Drug Discov.2010;9:615-627.

4. Bhatt I, Tripathi BN. Interaction of engineered nanoparticles with various components of the environment and possible strategies for their risk assessment. Chemosphere. 2011;82:308-317.

5. Chou CC1, Hsiao HY, Hong QS, et al. Single-walled carbon nanotubes can induce pulmonary injury in mouse model. Nano Lett. 2008; 8: 437-445.

6. Bartneck M1, Ritz T, Keul HA, et al. Peptide-functionalized gold nanorods increase liver injury in hepatitis. ACS Nano. 2012;23:8767-8777.

7. Schipper ML, Nakayama-Ratchford N, Davis CRet al. A pilot toxicology study of single-walled carbon nanotubes in a small sample of mice. Nat Nanotechnol. 2008; 3:216-221.

8. Wu J,Wang C,Sun J, Xue Y. Neurotoxicity of silica nanoparticles: Brain localization and dopaminergic neurons damage pathways. ACS Nano. 2011; 28: 4476-4489.

9. Lin $\mathrm{P}$, Chen JW, Chang LW, et al. Computational and ultrastructural toxicology of a nanoparticle, Quantum Dot 705, in mice. Environ Sci Technol. 2008;15:6264-6270

10. Bai Y, Zhang Y, Zhang J, et al. Repeated administrations of carbon nanotubes in male mice cause reversible testis damage without affecting fertility. Nat Nanotechnol. 2010;5: 683-689.

11. El-Sayed YS, Shimizu R, Onoda A Takeda K, et al. Carbon black nanoparticle exposure during middle and late fetal development induces immune activation inmale offspring mice. Toxicology. 2015; 2: 53-61.

12. Sun J1, Zhang Q, Wang Z, et al. Effects of nanotoxicity on female reproductivity and fetal development in animal models. Int J Mol Sci. 2013;29: 9319-9337.

13. Roychoudhury $S$, Nath $S$, Massanyi $P$, et al. Copper-induced changes in reproductive functions: in vivo and in vitro effects. Physiol Res. 2016; 65: 11-22.

14. Yamashita K, Yoshioka Y, Higashisaka K, Mimura K, et al. Silica and titanium dioxide nanoparticles cause pregnancy complications in mice. Nat Nanotechnol. 2011; 6: 321-328 .

15. Sumner SC, Fennell TR, Snyder RW, et al. Distribution of carbon-14 labeled C60 $([14 \mathrm{C}] \mathrm{C} 60)$ in the pregnant and in the lactating dam and the effect of $\mathrm{C} 60$ exposure on the biochemical profile of urine. J Appl Toxicol. 2010; 30: 354-360.

16. Semmler-Behnke M, Lipka J, Wenk A, et al. Size dependent translocation and fetal accumulation of gold nanoparticles from maternal blood in the rat. Part Fibre Toxicol. 2014; 11:33.

17. Huang JP, Hsieh PC, Chen CY, et al. Nanoparticles can cross mouse placenta and induce trophoblast apoptosis. Placenta. 2015; 36:1433-1441

18. Chattopadhyay S, Dash SK, Tripathy S, et al. Toxicity of cobalt oxide nanoparticles to normal cells; an in vitro and in vivo study. Chem Biol Interact. $2015 ; 25: 58-71$.

19. Adamcakova-Dodd A, Monick MM, Powers LS, et al. Effects of prenatal inhalation exposure to copper nanoparticles on murine dams and offspring. Part Fibre Toxicol. 2015; 12:30.

20. Nawaz M, Manzl C, Lacher V, et al. Copper-induced stimulation of extracellular signal-regulated kinase in trout hepatocytes: the role of reactive oxygen species, $\mathrm{Ca}^{2+}$, and cell energetics and the impact of extracellular signal-regulated kinase signaling on apoptosis and necrosis. Toxicol Sci. 2006; 92:464-475. 
21. Uauy R1, Maass A, Araya M. Estimating risk from copper excess in human populations. Am J Clin Nutr. 2008; 88:867S-871S.

22. Roychoudhury S, Massanyi P, Bulla J, et al. In vitro copper toxicity on rabbit spermatozoa motility, morphology and cell membrane integrity. J Environ Sci Health A Tox Hazard Subst Environ Eng. 2010; 45:1482-1491 .

23. Roychoudhury S, Bulla J, Sirotkin AV, et al. In vitro changes in porcine ovarian granulosa cells induced by copper. J Environ Sci Health A Tox Hazard Subst Environ Eng. 2014;49:625-633.

24. Gomes SI, Murphy M, Nielsen MT, et al. Cu-nanoparticles ecotoxicity-explored and explained? Chemosphere. 2015; 139: 240-245.

25. Manna P1, Ghosh M, Ghosh J, et al. Contribution of nano-copper particles to in vivo liver dysfunction and cellular damage: role of $\mathrm{\kappa Ba} / \mathrm{NF}-\mathrm{kB}, \mathrm{MAPKs}$ and mitochondrial signal. Nanotoxicology. 2012; 6:1-21.

26. Sau TK, Rogach AL. Nonspherical noble metal nanoparticles: colloid-chemical synthesis and morphology control. Adv Mater. 2010; 22: 1781-1804 .

27. Kwon J, Park S, Haque MM, et al. Study of sintering behavior of vapor forms of 1-octanethiol coated copper nanoparticles for application to ink-jet printing technology. J Nanosci Nanotechnol. 2012; 12:3434-3437.

28. Chen Z1, Meng H, Xing G, et al. Acute toxicological effects of copper nanoparticles in vivo . Toxicol Lett. 2006; 25:109-120.

29. Sarkar A, Das J, Manna P, et al. Nano-copper induces oxidative stress and apoptosis in kidney via both extrinsic and intrinsic pathways. Toxicology. 2011;18:208-217.

30. Anjum NA, Adam V, Kizek R, et al. Nanoscale copper in the soil-plant system-toxicity and underlying potential mechanisms. Environ Res. 2015; 138: 306-325.

31. Yang B, Wang Q, Lei R, et al. Systems toxicology used in nanotoxicology: mechanistic insights into the hepatotoxicity of nano-copper particles from toxicogenomics. J Nanosci Nanotechnol. 2010; 10: 8527-8537.

32. Kaweeteerawat $\mathrm{C}$, Chang $\mathrm{CH}$, Roy KR, et al. $\mathrm{Cu}$ Nanoparticles Have Different Impacts in Escherichia coli and Lactobacillus brevis than Their Microsized and Ionic Analogues. ACS Nano. 2015; 28:7215-7225.

33. Michaluk A, Kochman K. Involvement of copper in female reproduction. Reprod Biol. 2007; 7: 193-205.

34. Beker van Woudenberg A, Gröllers-Mulderij M, Snel C, et al. The bovine oocyte in vitro maturation model: a potential tool for reproductive toxicology screening. Reprod Toxicol. 2012; 34: 251-260.

35. Gao G, Ze Y, Li B, et al. Ovarian dysfunction and gene-expressed characteristics of female mice caused by long-term exposure to titanium dioxide nanoparticles. J Hazard Mater. 2012; 243: 19-27.

36. Chen XY, Chen WL, Ma M, et al. The potential of follicle-stimulating hormone peptide-modified triptolide-loaded nanoparticles to induce a mouse model of premature ovarian insufficiency . Int J Nanomedicine. 2015; 7: 2765-2774.

37. Yang $\mathrm{H}, \mathrm{Du} \mathrm{L}$, Tian $\mathrm{X}$, et al. Effects of nanoparticle size and gestational age on maternal biodistribution and toxicity of goldnanoparticles in pregnant mice. Toxicol Lett. 2014; 1: 10-18.

38. Stelzer R, Hutz RJ. Gold nanoparticles enter rat ovarian granulosa cells and subcellular organelles, and alter in-vitro estrogen accumulation. J Reprod Dev. 2009; 5: 5685-5690.

39. Gasperowicz M, Otto F. The notch signalling pathway in the development ofthe mouse placenta. Placenta. 2008; 29: 651-659.

40. Lam $\mathrm{C}$, Lim $\mathrm{KH}$, Karumanchi SA. Circulating angiogenic factors in the pathogenesis and prediction of preeclampsia. Hypertension. 2005; 46: 1077-1085.

41. Massimiani M, Salvi S, Piccirilli D, et al. Epidermal growth factor-like domain 7 promotes migration and invasion of human trophoblast cells through activation of MAPK, PI3K and NOTCH signaling pathways. Mol Hum Reprod. 2015; 21:435-451.

42. AshaRani PV, Low Kah Mun G, et al. Cytotoxicity and genotoxicity of silver nanoparticles in human cells ACS Nano. ACS Nano. 2009; 24: 279-290.

43. Kumar R, Dwivedi PD, Dhawan A, et al. Citrinin-generated reactive oxygen species cause cell cycle arrest leading to apoptosis via the intrinsic mitochondrial pathway in mouse skin. Toxicol Sci. 2011; 122: 557-566.

44. Stensberg MC, Madangopal R, Yale G, et al. Silver nanoparticle-specific mitotoxicity in Daphnia magna . Nanotoxicology. 2014; 8: 833-842 .

45. Park GB, Kim YS, Lee HK, et al. Reactive oxygen species and p38 MAPK regulate Bax translocation and calcium redistribution in salubrinal-induced apoptosis of EBV-transformed B cells. Cancer Lett. 2011; 27: 235-248

46. Yang CR, Liao WS, Wu YH, et al. CR108, a novel vitamin K3 derivative induces apoptosis and breast tumor inhibition by reactive oxygen species and mitochondrial dysfunction. Toxicol Appl Pharmacol. 2013; 15: 611-622.

47. Luo C, Li Y, Yang L, et al. Activation of Erk and p53 regulates copper oxide nanoparticle-induced cytotoxicity in keratinocytes and fibroblasts. Int J Nanomedicine. 2014; 10: 4763-4772.

48. Wang H, Jiang D, Liu J, et al. Compound $\mathrm{K}$ induces apoptosis of bladder cancer T24 cells via reactive oxygen species-mediated p38 MAPK pathway. Cancer Biother Radiopharm. 2013; 28: 607-614. 